\title{
Implementation of Conductance Tomography in Detection of the Hall Sensors Inhomogeneity
}

\author{
O. PetruK ${ }^{a, *}$, P. NOWAK ${ }^{b}$ AND R. SzeWCZYK ${ }^{b}$ \\ ${ }^{a}$ Industrial Research Institute for Automation and Measurements, Al. Jerozolimskie 202, 02-486 Warsaw, Poland \\ ${ }^{b}$ Institute of Metrology and Biomedical Engineering, Warsaw University of Technology, \\ sw. A. Boboli 8, 02-525 Warsaw, Poland
}

\begin{abstract}
Tomography is a useful tool for objects reconstruction in non-destructive testing. Many kinds of tomography, depending on the penetrating wave character, are available and adapted for specific application. This paper presents new kind of tomography - conductance tomography extended with a Hall effect. Its development was motivated by the need on inhomogeneity detection in thin film Hall effect sensor, particularly graphene Hall effect sensors. Paper presents complete description of the tomographic method and tomography software developed in the GNU Octave. Inverse transformation is based on optimization method. Each shape reconstruction was done with the finite element method using the open source software: Elmer FEM and Salome. Results confirmed the suitability of the work
\end{abstract}

DOI: 10.12693/APhysPolA.131.1186

PACS/topics: 07.55.Ge, 85.75.Ss, 85.30.Fg, 02.70.Dh

\section{Introduction}

The Hall-effect sensors are one of the most commonly used magnetic field sensors. Due to the utilization of advanced electronic materials, their operational parameters are constantly improving. On the other hand, new problems arise with the usage of a new materials and manufacturing technologies. Among industrially produced sensors dominate those made in thin-layer technology. This technology results with the presence of defects in the sensors structure, which causes significant ohmic asymmetry of the structure. Thus proper selection procedure is required. Structures with significant imbalance are rejected, which decreases manufacture process yield. Commonly known methods of the Hall-effect sensors imbalance compensation are based on external resistors [1], external conditioning circuit $[2,3]$ or by specific connections of the sensors leads [4].

Alternative solution for imbalance problem is a correction of the sensors structure by a removal of selected area [5] (e.g. laser etching similarly to the laser correction of the resistors). Till now usage of this method was limited due to lack of methods for determination of correction area.

Paper describes usage of resistance tomography for the correction area determination which may be a breakthrough in Hall-effect sensors adjustment. New correction method of thin-layer Hall-effect sensors (e.g. graphene based) [6] is presented. Another advantage of presented method is possibility for estimation of ohmic asymmetry reduction and resulting offset voltage. Novelty also applies to the method of correction areas deter-

\footnotetext{
*corresponding author; e-mail: opetruk@piap.pl
}

mination and dedicated layout of the Hall-effect sensor structure.

\section{Measurement setup}

In order to implement new method of the Hall-effect sensors compensation, new structure layout of the sensor, especially dedicated for resistance tomography, was designed.

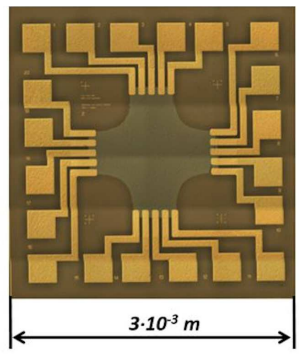

a)

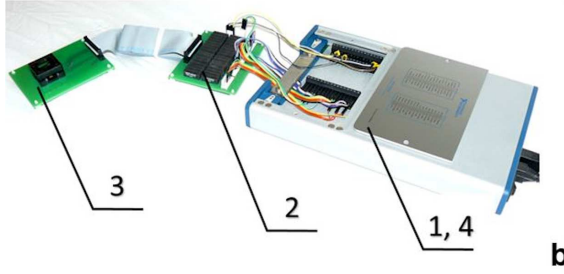

b)

Fig. 1. Measurement methodology: (a) new Hall-effect sensors structure, (b) measurement setup: 1 - power supply module, 2 - muliplexer; 3 - measuring socket, 4 - data acquisition module.

Proposed solution is based on increased number of electrodes. Instead of typically used 4 electrodes, new Hall-effect sensors structure contains separated contacts, where each traditional contact was multiplied as presented in Fig. 1a. Due to the technological limits, single 
sensor terminal now has 5 electrodes. Resistance measurement between the electrodes is conducted by specially designed resistance tomography setup and is based on technical method. As presented in Fig. 1b the setup contains 4 modules: power supply module, multiplexer, voltmeter and measuring socket dedicated for Hall-effect sensors in QFN casing. As a result of tomography measurements resistance matrix (1) is obtained. It contains values of resistance between each pair of the sensors electrodes

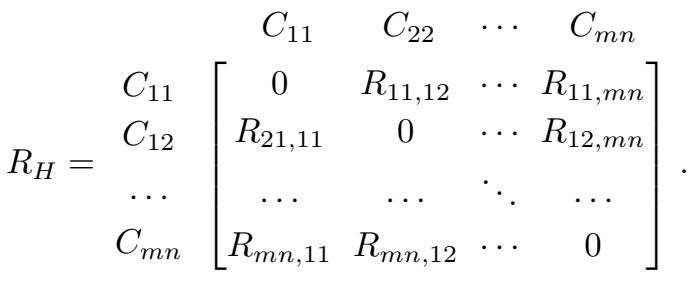

\section{Tomography transformation}

Resistance matrix obtained through the tomography measurement allows to use the tomography transformation for determination of location of sensors structure. Conductance distribution of sensitive structure in thinlayer sensors should be homogeneous. However, it can be disturbed by structure defects. Thus inverse tomography transformation in this case consists of determination of areas with nominal conductivity and areas with zero conductivity, which significantly differs with continous conductance distribution obtained in traditional resistance tomography $[7,8]$.

Inverse tomography transformation was based on iterative algorithm, where in each step shape reconstruction is optimized as presented in Fig. 2.

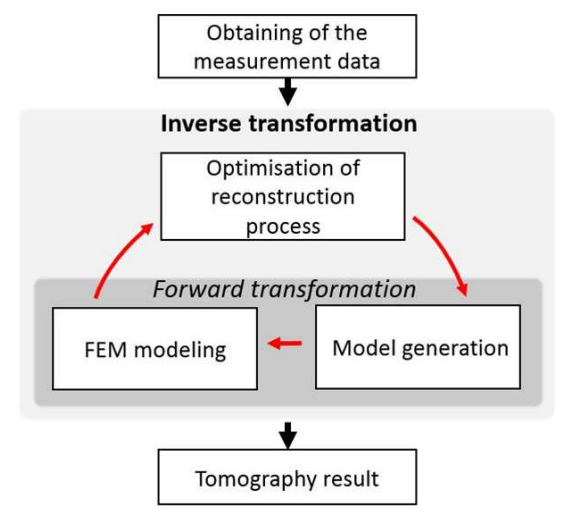

Fig. 2. Inverse tomography transformation algorithm.

Two optimization algorithms were implemented. None of them requires formulation of implied derivative of optimized function:

- the Nelder-Mead algorithm,

- the Levenberg-Marquardt algorithm.

Developed forward tomography transformation is based on Elmer FEM open-source software which utilizes finite element method. Shape reconstruction, for the Halleffect sensors compensation, is implemented in Octave open-source software.

\section{Validation of the method}

Presented method of the Hall-effect sensors compensation by its structure correction is based on identification of structure damage by a resistance tomography and then applying symmetrical structure correction with the usage of laser or other available methods. Usefulness of the method was verified on the numerical model presented in Fig. 3. During exemplary validation offset voltage was fivefold reduced - from $53.4 \mathrm{mV}$ to $9 \mathrm{mV}$ (with $1 \mathrm{~V}$ supply voltage).

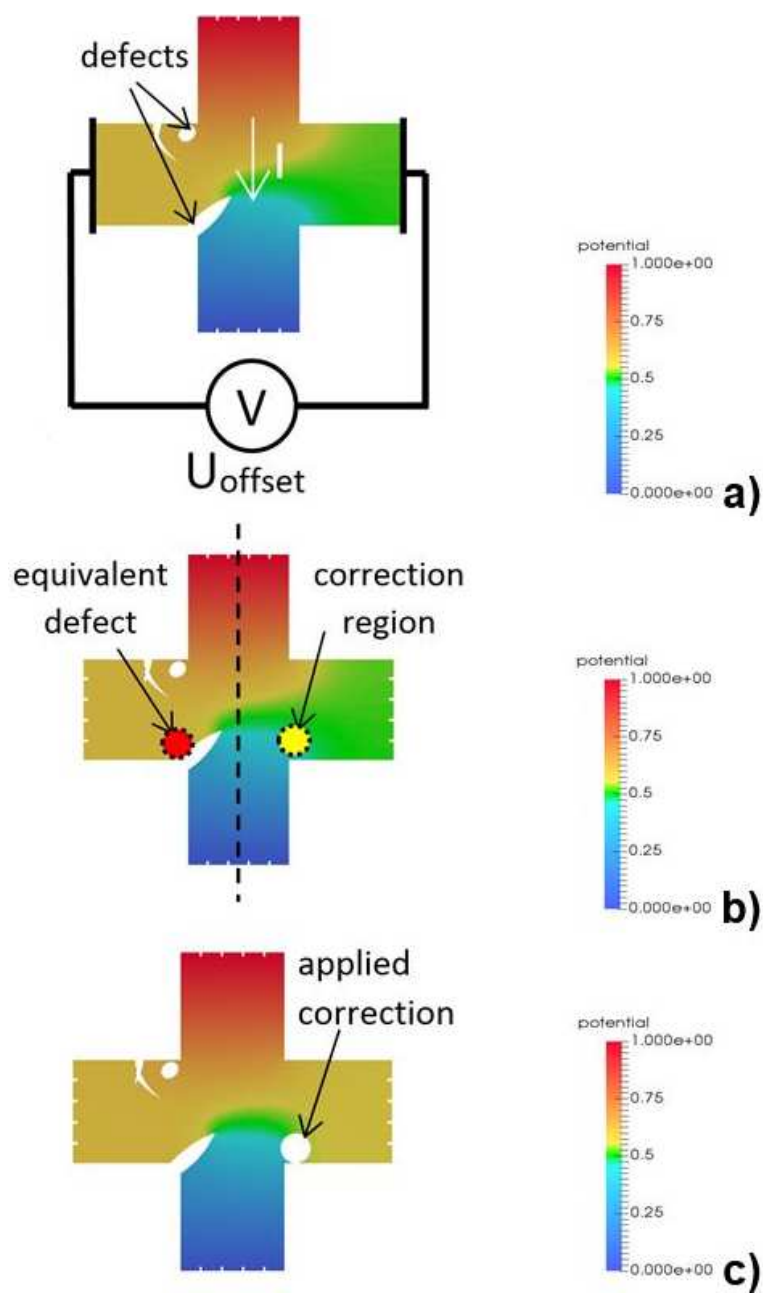

Fig. 3. Exemplary of correction of Hall-effect sensors structure. FEM validation of potential distribution: (a) sensors structure with simulated defect, (b) determination of corrective area on the basis of resistance tomography, (c) sensor after correction process.

\section{Conclusions}

Obtained results confirm usefulness of the usage of resistance tomography for correction of the Hall-effect sensors. During exemplary validation voltage offset was reduced 5 times. Very important, for the practical usage, is a possibility of prediction of correction result. 
New layout of the Hall-effect sensor which is suitable for resistance tomography was proposed. Dedicated setup for the tomography was designed and developed as well as software for tomography transformation. It needs to be highlighted that all components of developed software are open-source.

Due to the fact that laser correction of resistors is commonly used, it can be concluded that similar method for Hall-effect sensors may succeed commercially.

\section{Acknowledgments}

This work was partially supported by the statutory funds of Institute of Metrology and Biomedical Engineering, Warsaw University of Technology (Poland).

\section{References}

[1] J. Korvink, O. Paul, MEMS: A Practical Guide to Design, Analysis and Applications, William Andrew, New York 2005.
[2] H. Huang, D. Wang, Y. Xu, Sensors 15, 27359 (2015).

[3] A. Udo, Sensors, 2004, Proc. IEEE 3, (2004).

[4] M. Kachniarz, O. Petruk, R. Szewczyk, in: Advances in Intelligent Systems and Computing, Vol. 440, Eds. R. Szewczyk, C. Zielinski, M. Kaliczyńska, Springer, Berlin 2016, p. 763.

[5] S. Tumanski, Handbook of Magnetic Measurements, CRC Press, 2011.

[6] O. Petruk, R. Szewczyk, in: Advances in Intelligent Systems and Computing, Vol. 267, Eds. R. Szewczyk, C. Zielinski, M. Kaliczyńska, Springer, Berlin 2014, p. 631.

[7] A. Cultrera, L. Callegaro, in: 2015 IEEE 1st Int. Forum on Research and Technologies for Society and Industry Leveraging a better tomorrow (RTSI), Turin (Italy) 2015, p. 297.

[8] M.V.P. Kruger, K. Poolla, C.J. Spanos, in: Proc. 2002 American Control Conf. (IEEE Cat. No.CH37301), Anchorage (AK) 2002, p. 3678. 